\title{
Association between neurosarcoidosis with autonomic dysfunction and anti-ganglionic acetylcholine receptor antibodies
}

\author{
Makoto Oishi ${ }^{1} \cdot$ Akihiro Mukaino $^{1,2}\left(\right.$ M $\cdot$ Misako Kunii $^{3} \cdot$ Asami Saito $^{3} \cdot$ Yukimasa Arita $^{4} \cdot$ Haruki Koike $^{5}$. \\ Osamu Higuchi ${ }^{6} \cdot$ Yasuhiro Maeda $^{1} \cdot$ Norio Abiru $^{7} \cdot$ Naohiro Yamaguchi $^{8} \cdot$ Hiroaki Kawano $^{9} \cdot$ Eiko Tsuiki $^{10}$. \\ Tomonori Tanaka $^{11}$ - Hidenori Matsuo ${ }^{6}$. Masahisa Katsuno ${ }^{5} \cdot$ Fumiaki Tanaka $^{3}$ - Akira Tsujino ${ }^{1}$. Shunya Nakane ${ }^{2}$
}

Received: 25 December 2020 / Revised: 1 April 2021 / Accepted: 5 April 2021 / Published online: 21 April 2021

(c) The Author(s) 2021

\begin{abstract}
Objective To determine whether autonomic dysfunction in neurosarcoidosis is associated with anti-ganglionic acetylcholine receptor ( $\mathrm{gAChR}$ ) antibodies, which are detected in autoimmune autonomic ganglionopathy.

Methods We retrospectively extracted cases of sarcoidosis from 1787 serum samples of 1,381 patients between 2012 and 2018. Anti-gAChR antibodies against the $\alpha 3$ and $\beta 4$ subunit were measured by luciferase immunoprecipitation to confirm the clinical features of each case. We summarized literature reviews of neurosarcoidosis with severe dysautonomia to identify relevant clinical features and outcomes.

Results We extracted three new cases of neurosarcoidosis with severe dysautonomia, among which two were positive for anti-gAChR antibodies: Case 1 was positive for antibodies against the $\beta 4$ subunit, and Case 2 was positive for antibodies against both the $\alpha 3$ and $\beta 4$ subunits. We reviewed the cases of 15 patients with neurosarcoidosis and severe dysautonomia, including the three cases presented herein. Orthostatic hypotension and orthostatic intolerance were the most common symptoms. Among the various types of neuropathy, small fiber neuropathy (SFN) was the most prevalent, with seven of nine cases exhibiting definite SFN. Six of eight cases had impaired postganglionic fibers, of which the present three cases revealed abnormality of ${ }^{123}$ I-MIBG myocardial scintigraphy. Of the 11 cases, 10 were responsive to immunotherapy, except one seropositive case (Case 2).
\end{abstract}

Conclusions The presence of gAChR antibodies may constitute one of the mechanisms by which dysautonomia arises in neurosarcoidosis.

Keywords Neurosarcoidosis $\cdot$ Autonomic dysfunction $\cdot$ Anti-ganglionic acetylcholine receptor antibodies $\cdot$ Small fiber neuropathy

Akihiro Mukaino

a.mukaino@gmail.com

1 Department of Neurology and Strokology, Nagasaki University Hospital, Nagasaki, Japan

2 Department of Molecular Neurology and Therapeutics, Kumamoto University Hospital, 1-1-1, Honjo, Chuo-ku, Kumamoto 860-8556, Japan

3 Department of Neurology and Stroke Medicine, Yokohama City University Graduate School of Medicine, Yokohama, Japan

4 Department of Neurology, Matsuyama Red Cross Hospital, Ehime, Japan

5 Department of Neurology, Nagoya University Graduate School of Medicine, Nagoya, Japan

6 Department of Clinical Research, Nagasaki Kawatana Medical Center, Nagasaki, Japan
7 Department of Endocrinology and Metabolism, Unit of Advanced Preventive Medical Sciences, Division of Advanced Preventive Medical Sciences, Nagasaki University Graduate School of Biomedical Sciences, Nagasaki, Japan

8 Department of Psychiatry, Nagasaki University Hospital, Nagasaki, Japan

9 Department of Cardiology, Nagasaki University Hospital, Nagasaki, Japan

10 Department of Ophthalmology, Nagasaki University Hospital, Nagasaki, Japan

11 Department of Pathology, Nagasaki University Hospital, Nagasaki, Japan 


\section{Introduction}

Sarcoidosis presents with multiple non-caseating granulomas throughout the body, and neurological symptoms are thought to occur in $5-13 \%$ of the patients $[1,2]$. Approximately $15 \%$ of patients with neurosarcoidosis develop peripheral neuropathy [3]. Small fiber neuropathy (SFN), characterized by sensory disturbance and autonomic failure due to damage to the myelinated $A \delta$ and unmyelinated $\mathrm{C}$ fibers, is particularly common and has been reported in $44 \%$ of patients with sarcoidosis, potentially decreasing their quality of life [4-6].

Autoimmune autonomic ganglionopathy (AAG) is a rare disease characterized by various autonomic symptoms. The ganglionic neuronal nicotinic acetylcholine receptor (gAChR), consisting of two $\alpha 3$ and three $\beta 4$ subunits, mediates fast synaptic transmission in all peripheral autonomic ganglia in the autonomic nervous system [7]. Antibodies against gAChR are detected in approximately $50 \%$ of patients with AAG. Although the clinical features of neurosarcoidosis with dysautonomia are similar to those of AAG, the exact mechanism by which these symptoms arise remains unclear.

Herein, we aimed to elucidate the relationship between neurosarcoidosis with autonomic dysfunction and the presence of anti-gAChR antibodies.

\section{Methods}

\section{Patients}

We examined 1,787 serum samples of 1,381 patients from teaching and general hospitals throughout Japan between January 2012 and August 2018. We detected serum gAChR $\alpha 3$ and $\beta 4$ antibodies using the Luciferase Immunoprecipitation System assay and retrospectively identified cases that fulfilled the diagnostic guidelines for sarcoidosis $[8,9]$. In the present study, antibody levels were expressed as an antibody index (AI), which was calculated as follows: $\mathrm{AI}=$ (measured value in the serum sample [in relative luminescence units (RLU)])/(cut-off value [in RLU]). The normal AI value, established based on data from healthy individuals, was $<1.0$. We used the criteria for SFN proposed by Lacomis et al. [10], who stated that the diagnosis of SFN consists of three components: (1) symptoms of peripheral paresthesia that are typically painful, (2) specialized electrodiagnostic testing (normal nerve conduction studies and electromyogram), and (3) pathological findings [decreased intra-epidermal nerve fiber density (IENFD)]; these criteria are used to classify SFN as possible (one item positive), probable (two items positive), or definite (three items positive). Clinical data were obtained by reviewing the case records at each hospital.

All patients provided written informed consent for the storage and use of their serum and clinical information for research purposes. The study was approved by the Human Ethics Committees at the Nagasaki Kawatana Medical Center and Kumamoto University Hospital (Japan) (approval number 2011-21 and 1281, respectively).

\section{Histological analysis of skin biopsy}

A 3-mm punch biopsy was performed under local anesthesia (1\% lidocaine) in the right lower abdomen and medial surface of the right lower leg. Then, $50-\mu \mathrm{m}$ thick sections were immunostained using anti-human PGP 9.5 rabbit polyclonal rabbit antibody (Bio-Rad formerly AbDserotec \#7863-0504, Hercules, CA), and horseradish peroxidase-conjugated antirabbit polyclonal immunoglobulin $\mathrm{G}$ secondary antibody (\#424,144, NICHIREI BIOSCIENCES INC, Tokyo, Japan). We counted the number of small fibers in the dermis.

\section{Literature review}

A systematic review of the literature was conducted following the Preferred Reporting Items for Systematic Reviews and Meta-Analyses (PRISMA) reporting guidelines [11]. We also used Google Scholar and PubMed for our search targeting relevant peer-reviewed articles that were published between September 1985 and March 2020 using the following medical subject heading terms: "sarcoidosis", "neurosarcoidosis", "autonomic", and "small fiber neuropathy". The reference lists were found in relevant papers and textbooks manually. We extracted and tabulated data, including age at the onset of sarcoidosis, sex, mode of onset, autonomic symptoms, site of lesion in dysautonomia, immunotherapy and clinical outcome. We compared the frequency of autonomic dysfunction among cases of neurosarcoidosis with severe dysautonomia and anti-gAChR antibody-positive AAG.

\section{Statistical analysis}

A commercially available statistical software (SigmaPlot®); SPSS, Inc., Chicago, IL, USA) was used to analyze the data. We compared the prevalence of symptoms and associated data between patients with neurosarcoidosis with dysautonomia and those with anti-gAChR antibody-positive AAG; each of the analysis was assumed to be independent. Normally distributed data in both groups were analyzed by the Student's t-test for continuous variables (age at diagnosis) and by the Chi-squared test for categorical variables. For 
all analyses, $\mathrm{P}<0.05$ was considered to reflect statistically significant differences between groups.

\section{Results}

\section{General sample characteristics}

Among 1,381 patients, 179 gAChR antibody-positive patients had comorbid diseases, of whom 53 patients (30\%) presented with other autoimmune diseases, including Sjögren's syndrome in 20 patients and Hashimoto's disease in 13 patients; $19(11 \%)$ presented with tumors [ovarian $(n=5)$, lung $(n=5)$, gastric $(n=3)$, prostate $(n=2)$, maxillary sinus $(n=1)$, and mediastinal $(n=1)$ tumors; thymoma $(n=1)$; and seminoma $(n=1)]$ [12].

\section{Case presentation}

We examined three new cases of neurosarcoidosis presenting with severe autonomic disturbance.

\section{Case 1}

A 64-year-old man was admitted to the hospital due to orthostatic dizziness and recurrent syncope for 3 months. He had been diagnosed with pulmonary sarcoidosis 2 years prior to admission and treated with oral prednisolone $(10 \mathrm{mg}$ per day). He exhibited autonomic symptoms including orthostatic hypotension, orthostatic intolerance, xerophthalmia, constipation, and impotence. The serum levels of angiotensin-converting enzyme (ACE) were elevated (40.4 U/L [normal range $21.4 \mathrm{U} / \mathrm{L}$ ]). The coefficient of variation of $\mathrm{R}-\mathrm{R}$ intervals (CV R-R) was low (1.09\% at rest). Iodine-123 $\left.{ }^{123} \mathrm{I}\right)$ and meta-iodobenzylguanidine $\left({ }^{123} \mathrm{I}-\mathrm{MIBG}\right)$ myocardial scintigraphy revealed a reduced heart-to-mediastinum $(\mathrm{H} / \mathrm{M})$ ratio (early 1.94 , delayed 1.70 [normal range $\geq 2.20$, respectively]) and increased washout rate (57.71\%) (Fig. 1a, b). Single photon emission computed tomography with ioflupane I-123 injection (DaTscan ${ }^{\mathrm{TM}}$ ) was normal. The patient was positive for anti-gAChR antibodies against $\beta 4$ (AI: 1.012).

\section{Case 2}

A 69-year-old man was admitted to the hospital due to recurrent syncope and urinary retention for 2 years. He had been experiencing constipation for 10 years. A year prior to presentation, he had been prescribed midodrine hydrochloride, amezinium metilsulfate, and droxidopa, which had improved syncope; however, orthostatic intolerance persisted. He was diagnosed with pulmonary, skin, and ocular sarcoidosis. On admission, he exhibited various autonomic symptoms, including orthostatic hypotension, supine hypertension, orthostatic intolerance (dizziness, syncope), alternating loose stools, and constipation, as well as urinary retention. He also complained of a burning sensation from the umbilicus to the lateral side of his lower leg and great toe. Fluorodeoxyglucose (FDG)-positron emission tomography demonstrated the accumulation of FDG in the hilar and mediastinal lymph nodes (Fig. 2a). ${ }^{123}$ I-MIBG myocardial scintigraphy revealed a reduced $\mathrm{H} / \mathrm{M}$ ratio (early 1.53 , delayed 1.44) and increased washout rate (34.4\%) (Fig. 1c, d). Sympathetic skin response induced by stimulation of the median nerve demonstrated a low amplitude. The patient was positive for anti-gAChR antibodies against both $\alpha 3$ and $\beta 4$ subunits (AI 1.787 and 1.118 , respectively). Sural nerve biopsies revealed inflammatory cellular infiltration around the epineurial small vessels (Fig. 3a, b) and intact myelinated fibers. The patient was treated with two courses of steroid pulse therapy followed by oral administration of $40 \mathrm{mg}$ prednisolone per day. Although ocular sarcoidosis improved, autonomic symptoms did not improve. Due to glaucoma, his dose of prednisolone was tapered by $5 \mathrm{mg}$ per day over a month to a final dose of $5 \mathrm{mg}$. Despite administration of intravenous immunoglobulin (IVIg), autonomic symptoms did not improve after 3 years.

\section{Case 3}

A 27-year-old man with a history of type 1 diabetes mellitus and extensive dysautonomia was admitted to the hospital. He exhibited diabetic retinopathy. Upon admission, physical examination revealed sinus tachycardia with a pulse rate of 109 beats $/ \mathrm{min}$. There were palpable enlargements of the lymph nodes on the left side of the neck and groin, and a nodule in the left testis. Neurological examination revealed paresthesia in the distal extremities and absent Achillestendon reflexes. The patient exhibited various autonomic symptoms, including orthostatic intolerance, dry eyes and mouth, dry skin, alternating loose stools and constipation, neurogenic bladder, and erectile dysfunction. The serum levels of calcium and ACE were normal, and glycated hemoglobin was moderately elevated $7.5 \%$ [normal range $6.2 \%]$ ). Antibodies against gAChR were absent. The total cerebrospinal fluid protein levels were slightly elevated (52 mg/dL [normal range $40 \mathrm{mg} / \mathrm{dl}$ ]); however, cell count, glucose, ACE, and immunoglobulin G index were normal. Nerve conduction examinations yielded no significant findings. Head-up tilt test results were consistent with neurally mediated syncope; systolic blood pressure dropped from 100 to $80 \mathrm{mmHg}$ under isoproterenol provocation. The CV R-R was extremely low $(1.18 \%$ and $1.11 \%$ at rest and deep inspiration, respectively). A thermoregulatory sweat test revealed reduced sweating (Fig. 1e). ${ }^{123}$ I-MIBG myocardial scintigraphy revealed a reduced H/M ratio (early 1.49, delayed 1.39) 


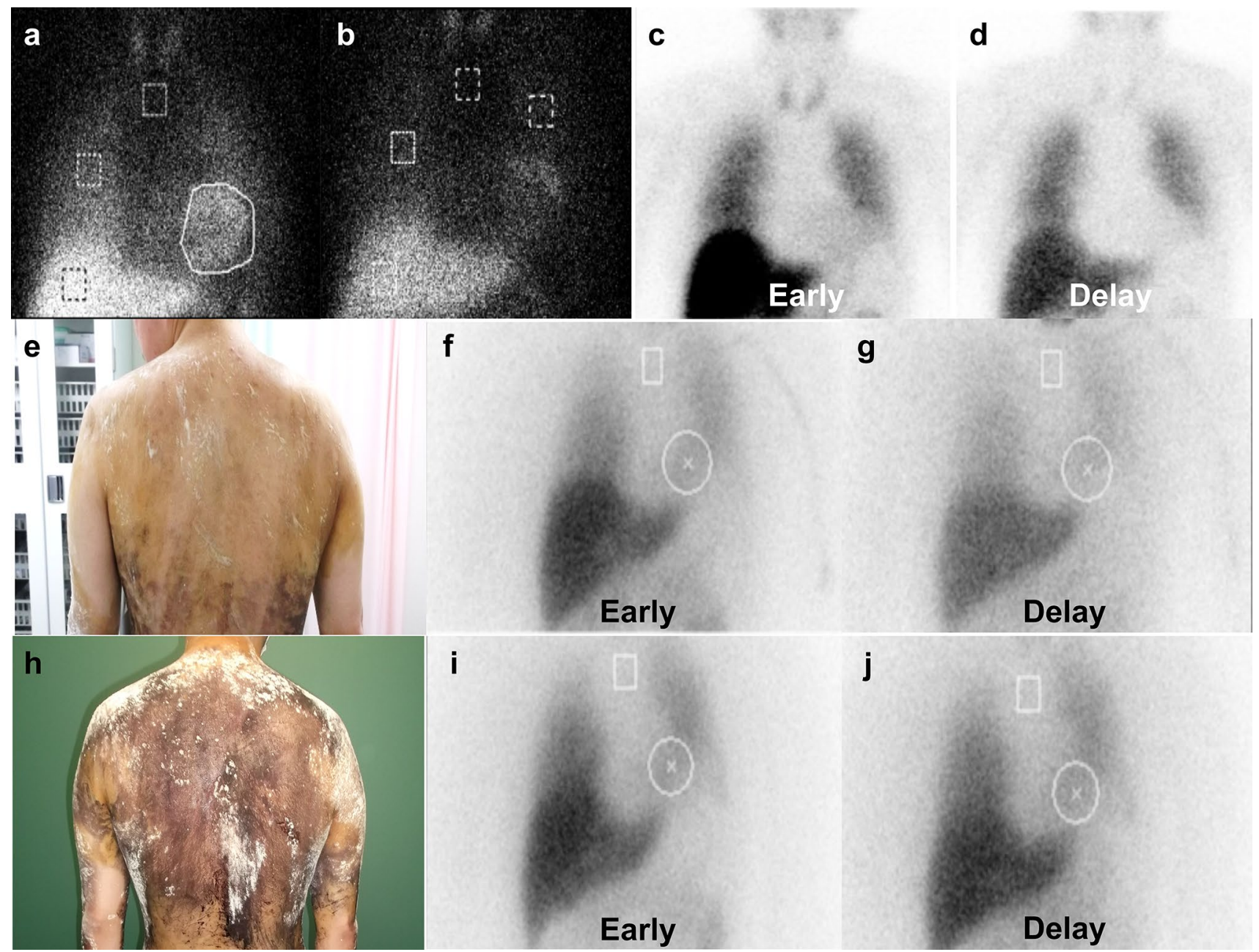

Fig. $1{ }^{123}$ I-MIBG myocardial scintigraphy and thermoregulatory sweating test. a-d, $\mathbf{f}, \mathbf{g}, \mathbf{i}, \mathbf{j}$ Iodine-123 and meta-iodobenzylguanidine myocardial scintigraphy of Cases 1-3. Note the reduced heart-tomediastinum $(\mathrm{H} / \mathrm{M})$ ratio: a early 1.94 , b delayed 1.70 in Case 1 ; $\mathbf{c}$ early 1.53 , $\mathbf{d}$ delayed 1.44 in Case 2 ; and $\mathbf{f}$, g pre-immunotherapy: $\mathbf{f}$ early 1.49 , g delayed 1.39 and increased washout rate (46.2\%) in Case

and increased washout rate (46.2\%; Fig. 1f, g). Contrastenhanced computed tomography demonstrated swollen hilar, mediastinal, supraclavicular fossa, cervical, axillary, lesser curvature, para-aortic, posterior pancreatico-duodenal, and inguinal lymph nodes; splenomegaly; multiple nodular lesions in the spleen; and an infiltrative, nodular shadow in the right lung. Gallium-67 scintigraphy revealed accumulations in the lacrimal and salivary glands, para-aortic and left groin lymph nodes, and left testis (Fig. 2b). Furthermore, gadolinium-enhanced brain magnetic resonance imaging revealed enlargement of the pituitary gland and hypothalamus. Inguinal lymph node biopsy revealed a non-caseating granuloma (Fig. 2c). We began oral administration of $60 \mathrm{mg}$ prednisolone per day. Surprisingly, the patient's autonomic symptoms improved dramatically. His constipation and
3. i, j Post-immunotherapy. Note the slight improvements in the H/M ratio: $\mathbf{i}$ early $1.66, \mathbf{j}$ delayed 1.63 and washout rate $(28.5 \%)$. e, $\mathbf{h}$ Thermoregulatory sweating test in Case 3. e Pre-immunotherapy. Note the reduced sweating. h Post-immunotherapy. Note that generalized sweating was apparent

light-headedness resolved; however, he continued to exhibit mild orthostatic tachycardia. His skin became less dry, and generalized sweating was apparent (Fig. 1h). ${ }^{123}$ I-MIBG myocardial scintigraphy revealed slight improvements in the $\mathrm{H} / \mathrm{M}$ ratio (early 1.66 , delayed 1.63 ) and washout rate (28.5\%; Fig. 1i, j). Decreasing the dosage of steroids did not cause a recurrence of symptoms; therefore, prednisolone treatment was reduced by $15 \mathrm{mg}$ per day after 9 months.

\section{Histological analysis of skin biopsy}

In Case 2, the skin biopsy from the right lower abdomen and medial surface of the right lower leg demonstrated a decrease in the dermal nerve fiber density (Supplementary Figure). 

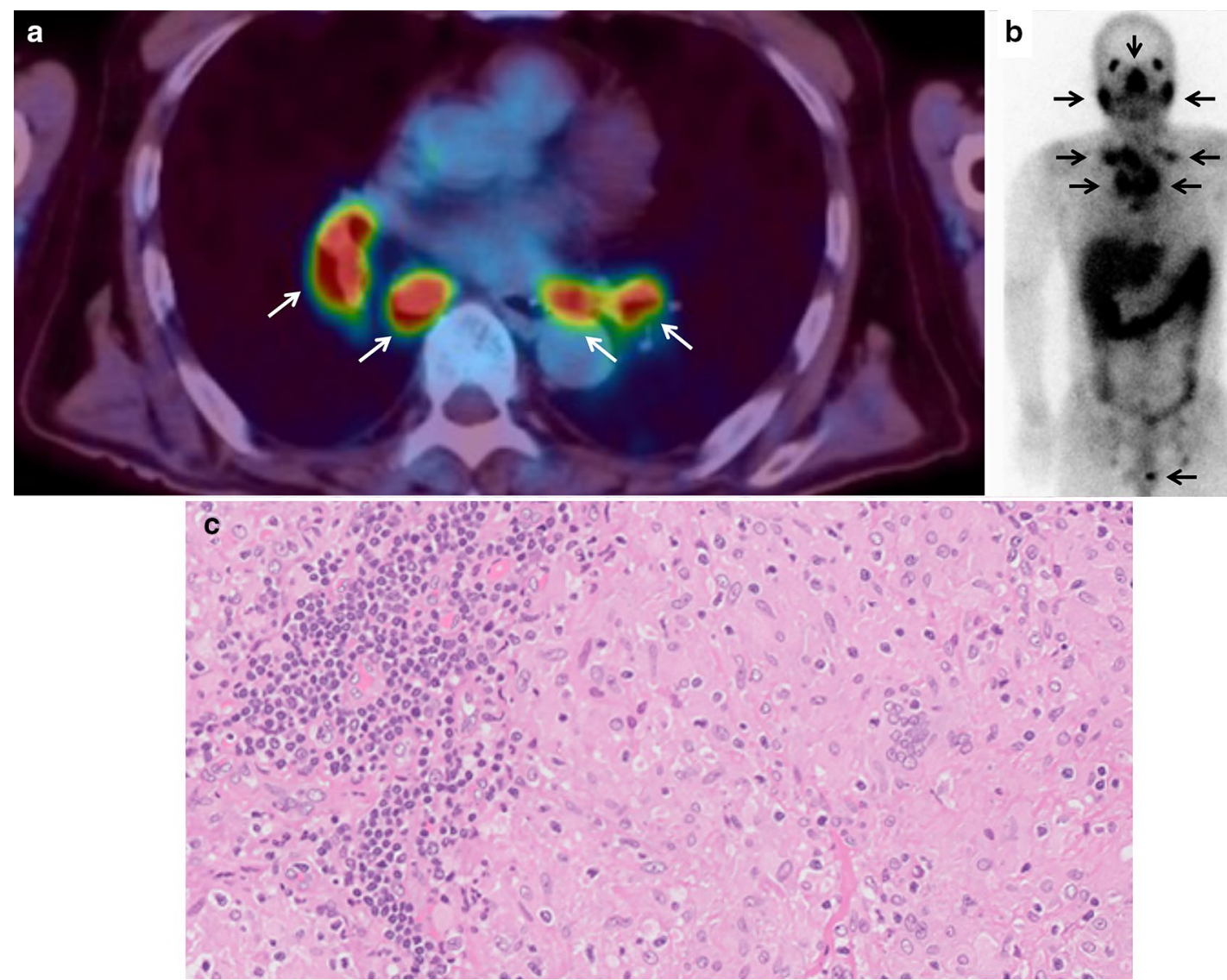

Fig. 2 Image and pathological findings of patients. a Fluorodeoxyglucose (FDG)-positron emission tomography of Case 2. Accumulation of FDG in the hilar and mediastinal lymph nodes. b Gallium-67 scintigraphy. Accumulations in the lacrimal and salivary glands, para- aortic and left groin lymph nodes, and left testis. c Inguinal lymph node biopsy. Non-caseating granuloma (hematoxylin-eosin staining, scale bar $=500 \mu \mathrm{m})$

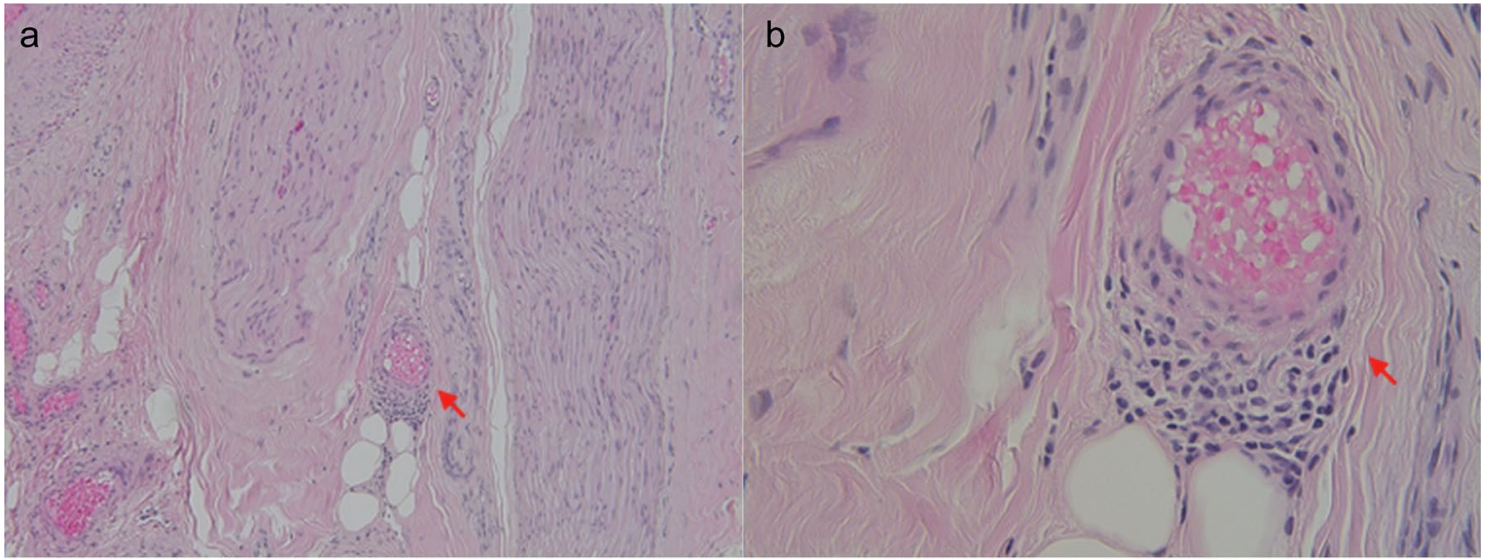

Fig. 3 Pathology of the sural nerve biopsy and skin biopsy of the abdomen and leg. a, b Sural nerve biopsy. Inflammatory cellular infiltration around epineurial small vessels. Arrow: inflammatory cell infiltration 


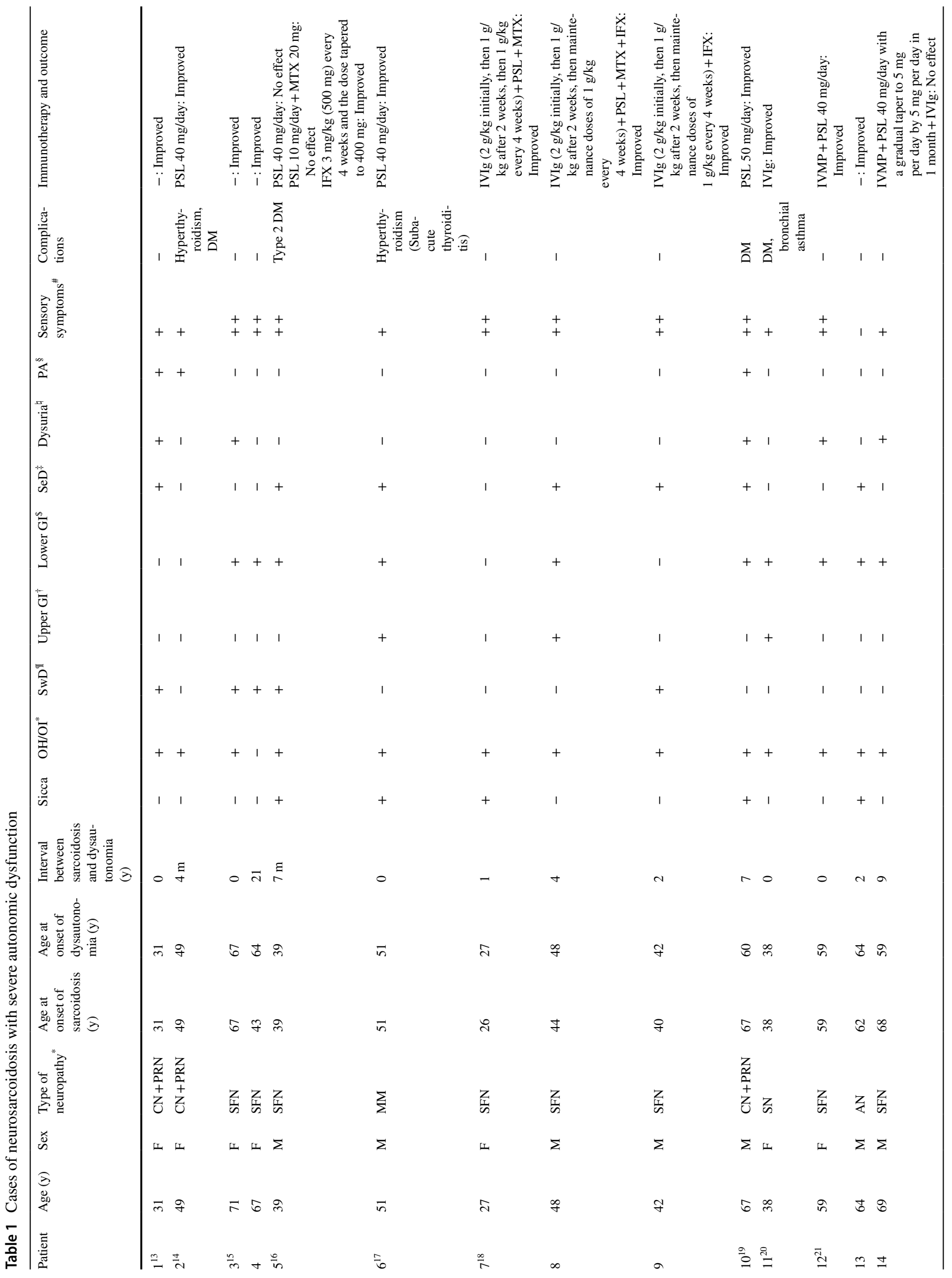




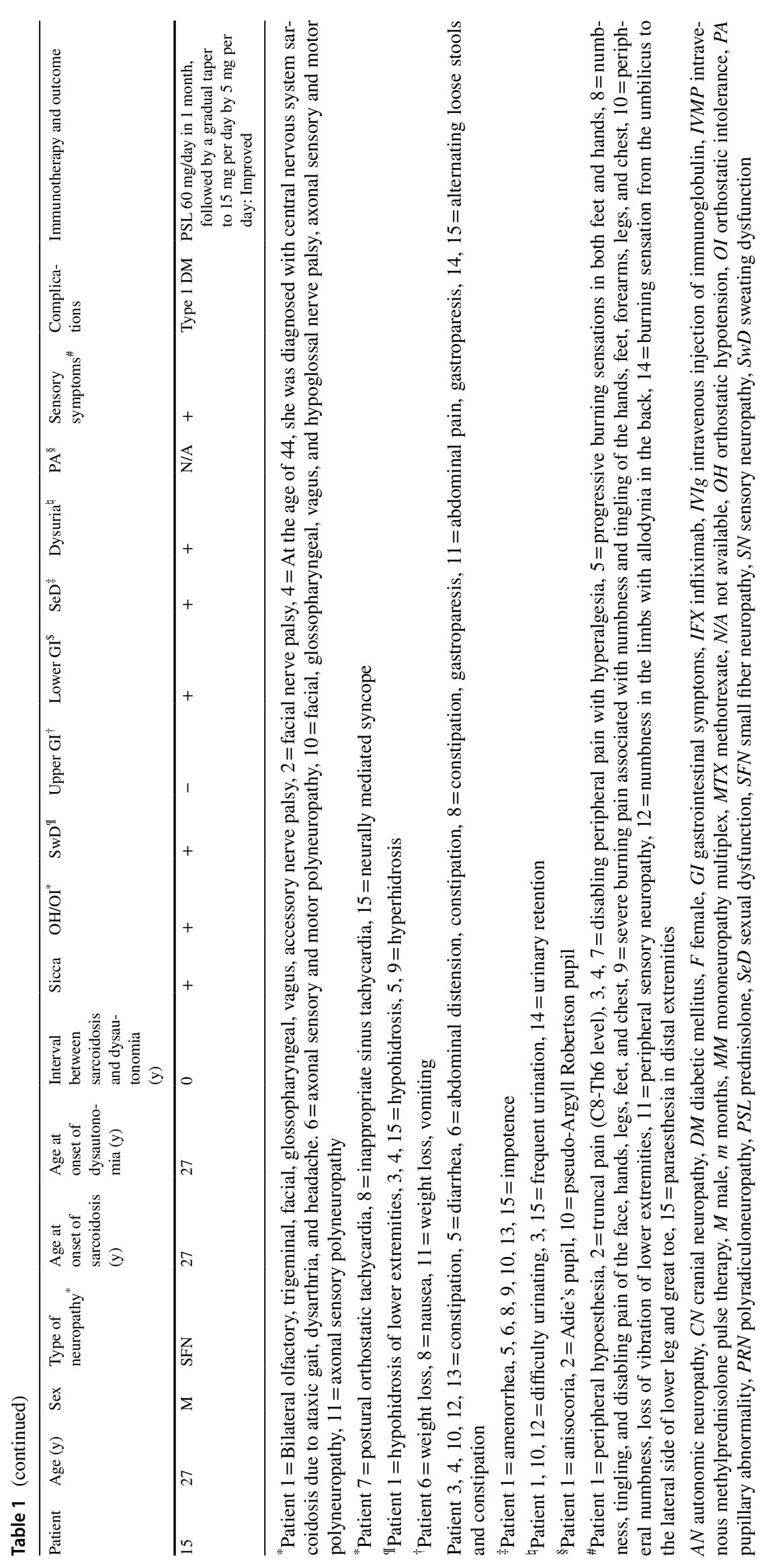




\section{Systematic literature review}

We obtained data for 15 cases of sarcoidosis with severe dysautonomia, including the three cases described above (designated as patients $13,14,15$, respectively, in Table 1) [13-21]. Out of the nine patients with SFN, seven had definite SFN (patients 3-5, 7-9, 12) and two (patients 14, 15) had probable SFN. The mean age at onset of sarcoidosis and dysautonomia was $47.4 \pm 14.0$ and $48.3 \pm 13.3$ years, respectively. All patients except patient 13 exhibited sensory symptoms, and eight patients (patients $3-5,7-10,12$ ) were severe cases, with symptoms including pain of burning quality, hyperalgesia, and allodynia (Fig. 4). Among the nine patients with SFN, four had length-dependent SFN (patients 3-5, 15) and five had non-length-dependent SFN/ ganglionopathy (patients 7-9, 12, 14). Immunotherapy was effective in all patients except patient 14.

The comparison of the frequency of autonomic dysfunction in neurosarcoidosis with severe dysautonomia and antigAChR antibody-positive AAG is shown in Table 2. Orthostatic hypotension and orthostatic intolerance were most common in both groups, followed by lower gastrointestinal symptoms. Patients with neurosarcoidosis with dysautonomia were significantly younger at diagnosis than those with
anti-gAChR antibody-positive AAG. Sexual dysfunction was significantly more frequent in neurosarcoidosis with dysautonomia than in anti-gAChR antibody-positive AAG.

The laboratory findings of sarcoidosis for the 15 patients are shown in Table 3. Hypergammaglobulinemia was observed in seven (patients 3, 6, 10-13, 15) of nine patients. Anti-gAChR antibodies were present in two of three patients (patients 13,14), of whom one was affected by probable SFN and the other by autonomic neuropathy.

The autonomic function test results for neurosarcoidosis with severe dysautonomia in six (patients 1, 6, 10, 13-15) of 15 patients who had undergone detailed examination are shown in Table 4. Patient 1 had a preganglionic disorder in the sympathetic nervous system, which was based on no response to $5 \%$ eye-drop solution of cocaine, a normal intradermal injection of acetylcholine despite reduced sweating in lower extremities, and increased plasma noradrenaline responsiveness upon postural change from supine to standing position. In the parasympathetic nervous system, the absence of supersensitivity to a $2.5 \%$ eyedrop solution of methacholine indicated no postganglionic impairment. Patient 6 had a postganglionic disorder in the sympathetic nervous system, which was based on no increase in plasma noradrenaline responsiveness upon
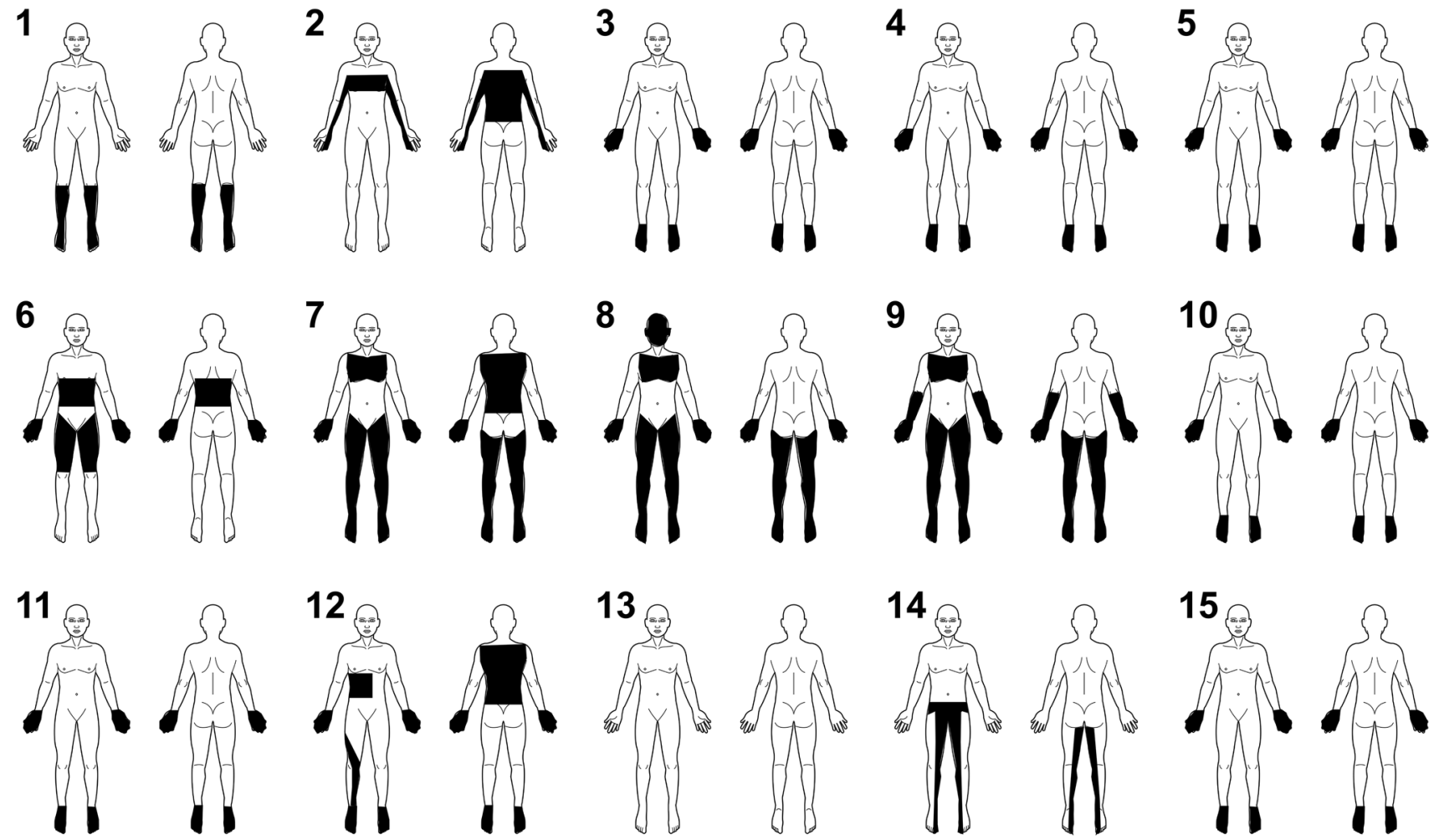

Fig. 4 Body diagrams representing the regional distribution of sensory symptoms in 15 patients. Patient $1=$ the legs and feet, $2=$ the C8-Th6 level and lower back; 3-5, 10, 11, 15 = the hands and feet; $6=$ the hands, Th4-11 level, and anterior region of thigh; $7=$ the

hands, chest, lower back, legs, and feet; $8=$ the face, hands, chest, legs, and feet; $9=$ the hands, forearms, chest, legs, and feet; $12=$ the hands, right Th5-11, back, right L4, and feet; $14=$ the umbilicus, lateral side of lower leg, and great toe 
Table 2 Characteristics of autonomic dysfunction in neurosarcoidosis with dysautonomia and anti-gAChR antibody-positive AAG

\begin{tabular}{llll}
\hline & $\begin{array}{l}\text { Neurosarcoidosis with } \\
\text { dysautonomia }\end{array}$ & $\begin{array}{l}\text { Anti-gAChR antibody- } \\
\text { positive AAG }\end{array}$ & P value \\
\hline Number of patients & 15 & 179 & .864 \\
Sex, number of male patients & $8(53.3 \%)$ & $106(59.0 \%)$ & $.031^{*}$ \\
Age at diagnosis (years) & $49.9 \pm 15.1$ & $59.0 \pm 20.0$ & .194 \\
OH & $14(93.3 \%)$ & $134(74.9 \%)$ & .480 \\
OI & $14(93.3 \%)$ & $148(82.7 \%)$ & .153 \\
Arrhythmia & $0(0 \%)$ & $32(21.1 \%)$ & .351 \\
Pupillary abnormalities & $3(20.0 \%)$ & $16(8.9 \%)$ & .902 \\
Sicca & $6(40.0 \%)$ & $81(45.3 \%)$ & .250 \\
Coughing & $0(0 \%)$ & $25(14.0 \%)$ & .710 \\
Anhidrosis & $6(40.0 \%)$ & $87(48.6 \%)$ & .191 \\
Upper GI dysfunction & $3(20.0 \%)$ & $73(40.8 \%)$ & .787 \\
Lower GI dysfunction & $11(73.3 \%)$ & $132(73.7 \%)$ & .146 \\
Bladder dysfunction & $5(33.3 \%)$ & $101(56.4 \%)$ & $<0.001 *$ \\
Sexual dysfunction & $8(100.0 \%)$ & $30(28.3 \%)$ & \\
\hline
\end{tabular}

$A A G$ autoimmune autonomic ganglionopathy, $g A C h R$ ganglionic acetylcholine receptor, GI gastrointestinal, $\mathrm{OH}$ orthostatic hypotension, $\mathrm{OI}$ orthostatic intolerance

Values are given as mean $\pm \mathrm{SD}$

$\mathrm{P}<0.05$ was considered statistically significant among the two groups (neurosarcoidosis with dysautonomia and anti-gAChR antibodies-positive AAG) postural change from supine to standing position, supersensitivity to a $1.25 \%$ eye-drop solution of adrenaline, and the absence of sympathetic skin response. In Patient 10, mydriasis and attenuated mydriasis were observed upon eye-drop solution of 5\% tyramine and 5\% cocaine administration, respectively, indicating preganglionic disturbance in the sympathetic nervous system. In addition, elevation in plasma noradrenaline concentration upon postural change from supine to standing position and the normal H/M ratio by MIBG myocardial scintigraphy suggested no sympathetic postganglionic impairment. In the parasympathetic nervous system, the absence of supersensitivity to eye-drop solution of $0.25 \%$ pilocarpine indicated no postganglionic impairment. Decreased sensation and voluntary inability to urinate, a large amount of residual urine, low bladder pressure, and atonic and involuntary pattern of contraction in bladder functional test indicated autonomous bladder. Spinal cord MRI showed no cauda equina lesions, suggesting parasympathetic preganglionic impairment. Patients 13 and 15 had postganglionic disorders in the sympathetic nervous system based on a reduced H/M ratio in ${ }^{123}$ I-MIBG myocardial scintigraphy. Patient 14 also had postganglionic disorders in the sympathetic nervous system based on a reduced H/M ratio in ${ }^{123}$ I-MIBG myocardial scintigraphy and low amplitude of sympathetic skin response. Patients 2 and 9 had postganglionic disorders based on Adie's pupil and abnormality in the quantitative sudomotor axon reflex test, respectively (data not shown in Table 4).

\section{Discussion}

We described three cases of neurosarcoidosis with dysautonomia, including anti-gAChR-positive cases. We also systematically reviewed the literature regarding neurosarcoidosis with dysautonomia.

Comparison of the frequency of autonomic dysfunction among patients with neurosarcoidosis with severe dysautonomia and those with anti-gAChR antibody-positive AAG revealed that orthostatic hypotension and orthostatic intolerance, followed by gastrointestinal symptoms, were the most common presentations in both groups. These results in neurosarcoidosis with severe dysautonomia are similar to those reported previously [22]. Further, they support similar autonomic symptoms' characteristics between neurosarcoidosis with autonomic dysfunction and AAG.

Hypergammaglobulinemia was observed in seven of nine patients. Hypergammaglobulinemia was previously reported in patients with sarcoidosis [23]. Moreover, it has been suggested that hypergammaglobulinemia or circulating immune complexes may be associated with the clinical course of sarcoidosis [23, 24]. A previous study also reported the presence of higher levels of B-cell activating factor, and correspondingly, higher numbers of IgG-producing B cells in the lung tissue [25]. The imbalance in the number of B cells and $\mathrm{T}$ follicular helper cell subsets is particularly important for antibody production and may be associated with development of sarcoidosis [26]. Patients with sarcoidosis frequently have higher titers of autoantibodies than healthy individuals [27-29]. Several autoantibodies, including 


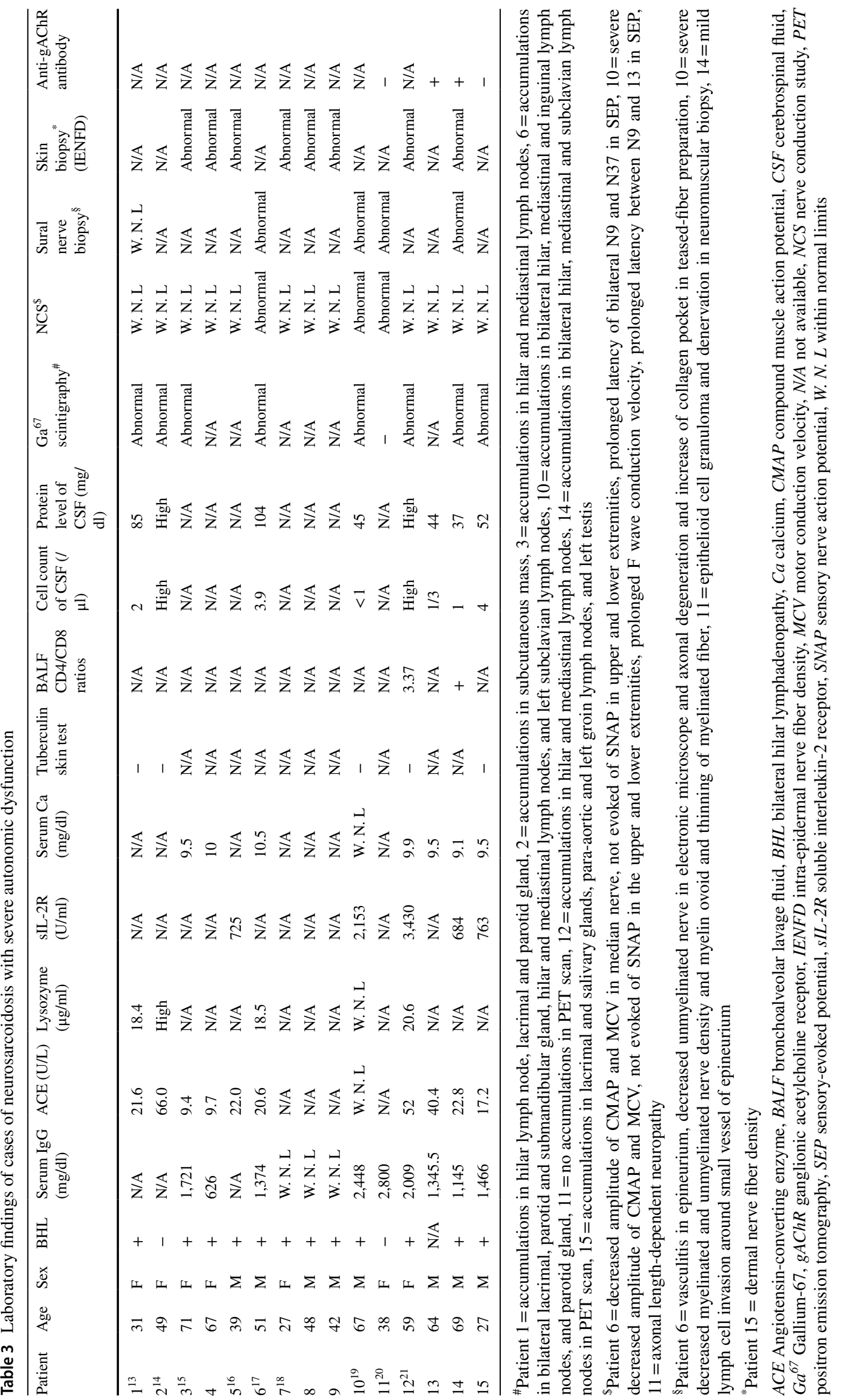




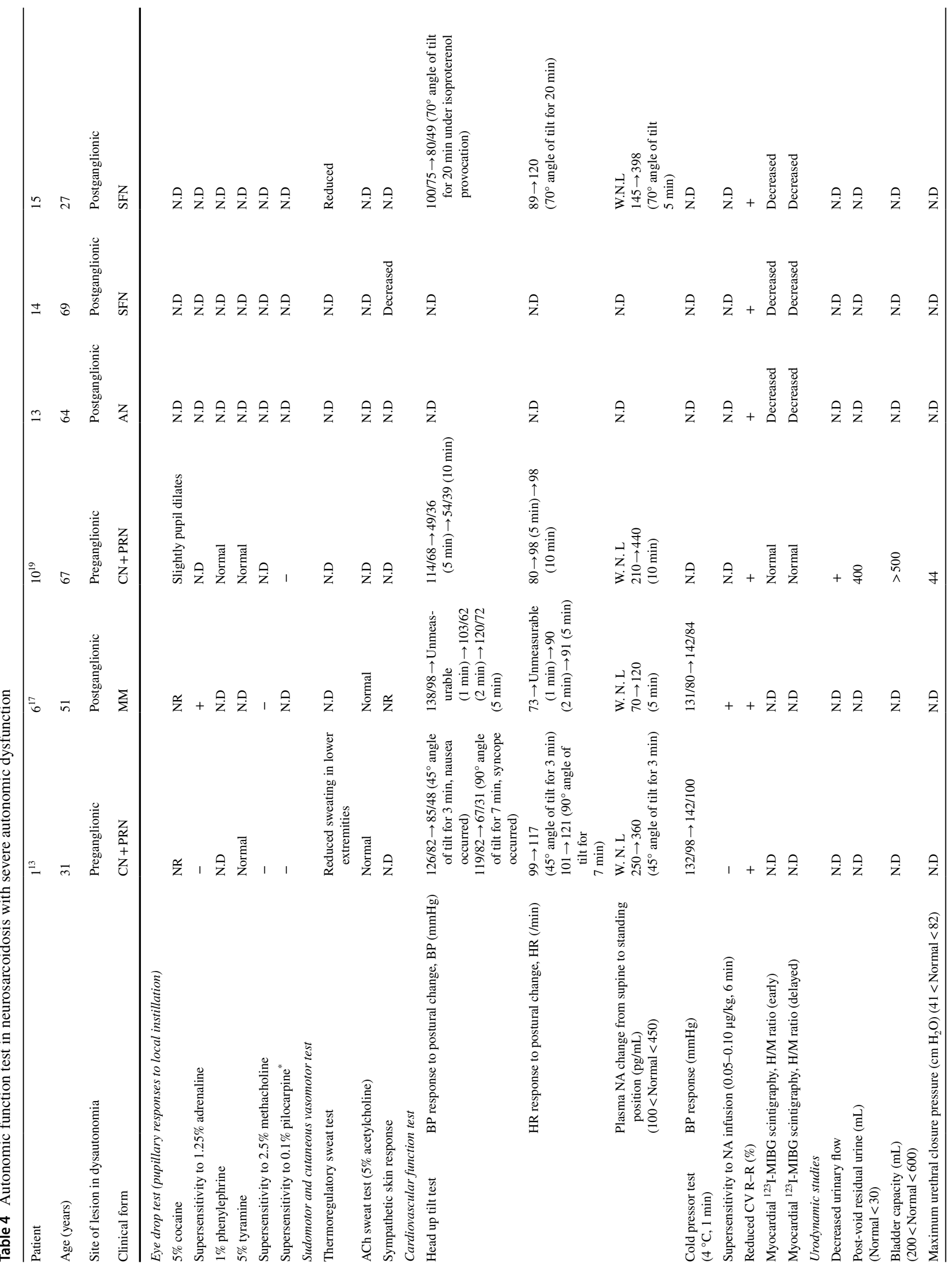




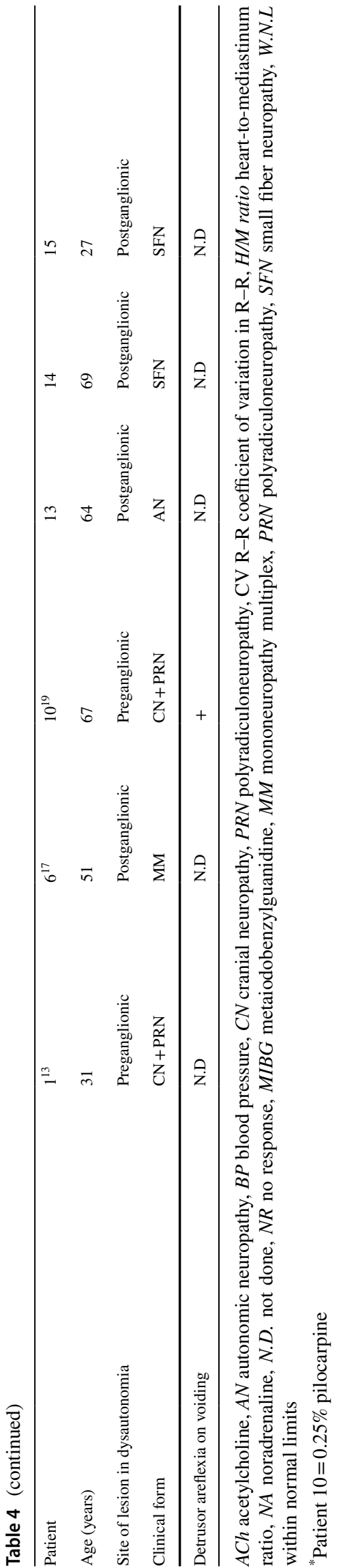

anti-mitochondrial, anti-nuclear, anti-double stranded DNA, anti-citrullinated cyclic peptide, rheumatoid factor, antivimentin, and anti-negative elongation factor $\mathrm{E}$, have been detected in sarcoidosis [27-34]. To the best of our knowledge, no previous study has reported anti-gAChR antibodies in sarcoidosis.

Among the presented cases and those in the reviewed literature, the most common type of neuropathy was SFN. There are two categories of SFN clinical symptoms: sensory symptoms such as neuropathic pain, allodynia, hyperalgesia, or pinprick and thermal sensory loss, and autonomic symptoms, including orthostatic intolerance, sicca symptoms, sweating disturbances, constipation, urinary disturbance, and sexual dysfunction. According to the self-reported burden, sarcoid-associated SFN (SSFN) was reported to be present in $86.2 \%$ of patients with sarcoidosis but may be overlooked by clinicians [35]. Patients with sarcoidosis with symptoms of SFN were found to have significantly decreased IENFDs as compared to patients with asymptomatic sarcoidosis and healthy individuals [36]. In our literature review, we focused on sarcoidosis with dysautonomia and found that many patients also have sensory symptoms, among whom seven patients were classified as definite SFN. Moreover, five were classified as non-length dependent SFN, one of whom was positive for anti-gAChR antibodies. Non-length dependent SFN is more commonly observed in immunemediated conditions than length-dependent SFN, and three IVIg-responsive cases of sarcoidosis affected by non-lengthdependent SFN have been described [37]. We previously reported a $46 \%$ rate of sensory disturbance among patients with anti-gAChR antibody-positive AAG [38], while a post-mortem study reported one case of a patient with nonlength-dependent SFN with nicotinic AChR antibodies [39]. The pathological mechanism of SSFN is known to involve proinflammatory cytokines, including tumor necrosis factor- $\alpha$ [16]. It was reported that patients with sarcoidosis carrying the HLA-DQB1*0602 allele are more likely to have abnormal temperature threshold testing, indicative of SFN, than do healthy individuals [40]. Although this result simply reflects a bystander effect in the pathogenesis of sarcoidosis, our cases suggest that anti-gAChR antibodies may contribute to the pathogenesis of SSFN.

At the site of lesion in dysautonomia, postganglionic denervation was more common, and we found reduced $\mathrm{H} / \mathrm{M}$ ratios and increased washout rates in ${ }^{123}$ I-MIBG myocardial scintigraphy in our three illustrative cases. Cardiac sympathetic dysfunction in sarcoidosis comorbid with SFN was previously demonstrated by ${ }^{123}$ I-MIBG myocardial scintigraphy [41]. Previous studies have revealed the presence of cardiac sympathetic dysfunction in up to $50 \%$ of patients with SSFN by ${ }^{123}$ I-MIBG myocardial scintigraphy [41, 42]. Importantly, anti-gAChR antibodies were detected in Cases 1 and 2 in our study. It has been demonstrated that 
a decreased $\mathrm{H} / \mathrm{M}$ ratio is observed in $80 \%$ of seropositive AAG cases, with several cases showing improvements in their $\mathrm{H} / \mathrm{M}$ ratio by immunotherapy $[8,38]$. In the present Case 3, the reduced H/M ratio in ${ }^{123}$ I-MIBG myocardial scintigraphy was improved by steroid therapy; therefore, it may have resulted from functional impairments in autonomic nerve fiber transmission. Autoantibodies against gAChR, which could have interfered with autonomic ganglionic synaptic transmission of the sympathetic and parasympathetic nerves, were not detected in this case. We speculate that other immunologic blocking factors, such as unknown antibodies or cytokines, may have caused the functional impairment in the postganglionic pathways.

Two patients (patients 1 and 10) presented with preganglionic autonomic dysfunction. Although it is not possible to determine whether the site of lesions in dysautonomia is the preganglionic fiber or the central nervous system strictly based on the results of the autonomic function test, we considered that the site of lesion of autonomic disturbance may be the spinal nerve root level. This was based on the fact that there was no loss of myelinated and unmyelinated fibers in sural nerve biopsy and that the levels of cerebrospinal fluid protein were elevated in patient 1 , while the $F$ wave conduction velocities in nerve conduction studies were prolonged in patient 10. Interestingly, both these patients had cranial neuropathy with polyradiculoneuropathy. We also consider that the preganglionic fiber disorder might have occurred as a partial symptom of sarcoid polyradiculoneuropathy. Regarding the distribution of sensory disturbance in the 15 literature cases, there were many cases in which peripheral nerves, including small fibers, were the site of lesion; however, in three cases of cranial neuropathy with polyradiculoneuropathy, the lesion site may have been the dorsal root. Eleven patients underwent immunotherapy, all of whom, except one (Case 2), were responsive to immunotherapy. While some patients with sarcoidosis-related autonomic dysfunction showed improvements after treatment with oral prednisolone alone, there were several intractable cases that were only improved after combining multiple therapeutic agents, such as intravenous methylprednisolone pulse, IVIg, prednisolone, and immunosuppressants. The seropositive patient (Case 2) did not show any improvement despite immunotherapy by steroid pulse, oral prednisolone, and IVIg. Past studies have suggested the use of a combined therapy consisting of steroids, plasmapheresis, or IVIg, followed by oral prednisolone and immunosuppressive agents, such as azathioprine, mycophenolate mofetil, and rituximab, in severe patients with AAG [8]. Therefore, we speculated that AAG might be occurring in combination with neurosarcoidosis in this case, and that a combination of immunotherapy other than steroids and IVIg may be necessary.

This study has several limitations. First, we could not follow-up on the patients after the treatment for potential recurrences. Second, five of the 15 cases had diabetes mellitus, which may have affected autonomic dysfunction. Based on the dramatic response to immunotherapy, we surmised that these cases of autonomic dysfunction may be caused by dysimmune mechanisms related to sarcoidosis, although coexisting underlying diabetic neuropathy cannot be excluded. Third, the present study included a small study population without appropriate control groups. Further, we could not confirm whether anti-gAChR antibodies were present in patients with normal sarcoidosis or neurosarcoidosis without autonomic dysfunction. Therefore, we plan to assess this in our future studies. Finally, we previously reported that the levels of anti-gAChR antibodies may not necessarily affect the severity of the disease among patients with anti-gAChR-positive AAG [12]. Further prospective studies involving a large number of patients are necessary to address the prevalence of anti-gAChR antibodies among patients with neurosarcoidosis with dysautonomia, assess the rates of responsiveness to immunotherapy depending on the presence or absence of anti-gAChR antibodies, and investigate potential treatment strategies.

\section{Conclusion}

We reported neurosarcoidosis cases with severe dysautonomia, cases that have tested positive for anti-gAChR antibodies. Anti-gAChR antibodies may be associated with the pathogenesis of dysautonomia in sarcoidosis. Further studies are needed to elucidate the relationship between neurosarcoidosis with dysautonomia and anti-gAChR antibodies, and prospective studies to investigate potential treatment strategies are warranted.

Author's contributions AM had full access to all of the data in the study and takes responsibility for the integrity of the data and the accuracy of the data analysis. AM, SN conceived and designed the study. MO, AM, MK, AS, YA, HK ${ }^{5}, \mathrm{OH}, \mathrm{YM}, \mathrm{NA}, \mathrm{NY}, \mathrm{HK}^{9}$, ET, TT acquired the data. MO, AM, $\mathrm{HK}^{5}, \mathrm{HM}, \mathrm{MK}, \mathrm{TF}, \mathrm{AT}, \mathrm{SN}$ analyzed and interpreted the data. All authors were involved in drafting the article or making critical revisions for important intellectual content, and all authors read and approved the submitted manuscript.

Funding Supported by the Ministry of Health, Labor, and Welfare, Japan, and the Ministry of Education, Culture, Sports, Science, and Technology of Japan (JSPS KAKENHI [Grant Numbers 19K17012 and 19H03549]).

Availability of data and material The data that support the findings of this study are available from the corresponding author upon reasonable request.

\section{Declarations}

Conflict of interest/competing interests The authors declare that they have no conflict of interest. 
Ethics approval The study was approved by the Human Ethics Committees at the Nagasaki Kawatana Medical Center and Kumamoto University Hospital (Japan) (approval number 2011-21 and 1281, respectively).

Open Access This article is licensed under a Creative Commons Attribution 4.0 International License, which permits use, sharing, adaptation, distribution and reproduction in any medium or format, as long as you give appropriate credit to the original author(s) and the source, provide a link to the Creative Commons licence, and indicate if changes were made. The images or other third party material in this article are included in the article's Creative Commons licence, unless indicated otherwise in a credit line to the material. If material is not included in the article's Creative Commons licence and your intended use is not permitted by statutory regulation or exceeds the permitted use, you will need to obtain permission directly from the copyright holder. To view a copy of this licence, visit http://creativecommons.org/licenses/by/4.0/.

\section{References}

1. Delaney P (1977) Neurologic manifestations in sarcoidosis: review of the literature, with a report of 23 cases. Ann Intern Med 87:336-345. https://doi.org/10.7326/0003-4819-87-3-336

2. Lower EE, Broderick JP, Brott TG, Baughman RR (1997) Diagnosis and management of neurological sarcoidosis. Arch Intern Med 157:1864-1868

3. Hoitsma E, Faber CG, Drent M, Sharma OP (2004) Neurosarcoidosis: a clinical dilemma. Lancet Neurol 3:397-407. https:// doi.org/10.1016/S1474-4422(04)00805-1

4. Hoitsma E, Marziniak M, Faber CG, Reulen JPH, Sommer C, De Baets M, Drent M (2002) Small fibre neuropathy in sarcoidosis. Lancet 359:2085-2086. https://doi.org/10.1016/s0140-6736(02) 08912-2

5. Tavee J (2018) Office approach to small fiber neuropathy. Cleve Clin J Med 85:801-812. https://doi.org/10.3949/ccjm.85a.17124

6. Tavee J, Culver D (2019) Non-organ manifestations of sarcoidosis. Curr Opin Pulm Med 25:533-538. https://doi.org/10.1097/MCP. 0000000000000597

7. Wang Z, Low PA, Vernino S (2010) Antibody-mediated impairment and homeostatic plasticity of autonomic ganglionic synaptic transmission. Exp Neurol 222:114-119. https://doi.org/10.1016/j. expneurol.2009.12.016

8. Nakane S, Higuchi O, Koga M, Kanda T, Murata K, Suzuki T, Kurono H, Kunimoto M, Kaida K, Mukaino A, Sakai W, Maeda Y, Matsuo H (2015) Clinical features of autoimmune autonomic ganglionopathy and the detection of subunit-specific autoantibodies to the ganglionic acetylcholine receptor in Japanese patients. PLoS ONE 10:e0118312. https://doi.org/10.1371/journal.pone. 011831

9. Hunninghake GW, Costabel U, Ando M, Baughman R, Cordier JF, du Bois R, Eklund A, Kitaichi M, Lynch J, Rizzato G, Rose C, Selroos O, Semenzato G, Sharma OP (1999) ATS/ERS/WASOG statement on sarcoidosis. American Thoracic Society/European Respiratory Society/World Association of Sarcoidosis and other Granulomatous Disorders. Sarcoidosis Vasc Diffuse Lung Dis 16:149-173

10. Lacomis D (2002) Small-fiber neuropathy. Muscle Nerve 26:173188. https://doi.org/10.1002/mus.10181

11. Moher D, Liberati A, Tetzlaff J, Altman DG; PRISMA Group (2009) Preferred reporting items for systematic reviews and metaanalyses: the PRISMA statement. PLoS Med 6:e1000097. https:// doi.org/10.1371/journal.pmed.1000097
12. Nakane S, Mukaino A, Higuchi O, Maeda Y, Takamatsu K, Yamakawa M, Watari M, Tawara N, Nakahara K, Kawakami A, Matsuo H, Ando Y (2020) A comprehensive analysis of the clinical characteristics and laboratory features in 179 patients with autoimmune autonomic ganglionopathy. J Autoimmun 108:102403. https://doi.org/10.1016/j.jaut.2020.102403

13. Kazahaya Y, Kita K, Nakano Y, Hirayama K (1985) Sarcoid polyradiculoneuropathy with autonomic dysfunctions. Rinsho Shinkeigaku 25:1017-1022

14. Otsuka H, Morikawa N, Mukae H et al (1987) A case of sarcoidosis with various neurological symptoms, discovered with subcutaneous nodules. Nihon Sarcoidosis/Nikugeseishikkan Gakkaizasshi (The Japanese Journal of Sarcoidosis and Other Granulomatous Disorders) 7:69-70 (in Japanese)

15. Ikeda Y, Yamaguchi T, Yamada Y, Shinohara T, Kono C, Aoyagi T, Amano H, Kijima M, Kurose N, Miyamoto K (2004) Two sarcoidosis cases of small fiber neuropathy. Nihon sarcoidosis/ nikugeseishikkan gakkaizasshi (The Japanese Journal of Sarcoidosis and Other Granulomatous Disorders) 24:65-69 (in Japanese)

16. Hoitsma E, Faber CG, van Santen-Hoeufft M, De Vries J, Reulen JP, Drent M (2006) Improvement of small fiber neuropathy in a sarcoidosis patient after treatment with infliximab. Sarcoidosis Vasc Diffuse Lung Dis 23:73-77

17. Kimura Y, Takeuchi M, Ota K, Uchiyama S, Iwata M (2007) A case of sarcoidosis with severe autonomic dysfunction. Tokyo Jyoshi Ika Daigaku zasshi suppl:99-104.

18. Parambil JG, Tavee JO, Zhou L, Pearson KS, Culver DA (2011) Efficacy of intravenous immunoglobulin for small fiber neuropathy associated with sarcoidosis. Respir Med 105:101-105. https://doi.org/10.1016/j.rmed.2010.09.015

19. Yabuuchi K, Okazaki T, Nakamura K, Hanaoka T, Kimura N, Mori T, Hirano T, Kumamoto T (2013) A male case aged 67 of neurosarcoidosis with severe autonomic dysfunction. Nihon Sarcoidosis/Nikugeseishikkan Gakkaizasshi (The Japanese Journal of Sarcoidosis and Other Granulomatous Disorders) 33:139-145 (in Japanese)

20. Grignano E, Mekinian A, Dubourg O, Dhote R, Fain O (2013) Dramatic response to intravenous immunoglobulins in dysautonomic neurosarcoidosis. J Clin Neurosci 20:1807-1808. https:// doi.org/10.1016/j.jocn.2013.02.016

21. Saito H, Yamaguchi T, Adachi Y, Yamashita T, Wakai Y, Saito K, Shinohara Y, Suzuki K, Yagihashi S, Terada J, Tatsumi K (2015) Neurological symptoms of sarcoidosis-induced small fiber neuropathy effectively relieved with high-dose steroid pulse therapy. Intern Med 54:1281-1286. https://doi.org/10. 2169/internalmedicine.54.3702

22. Tavee JO, Karwa K, Ahmed Z, Thompson N, Parambil J, Culver DA (2017) Sarcoidosis-associated small fiber neuropathy in a large cohort: Clinical aspects and response to IVIG and antiTNF alpha treatment. Respir Med 126:135-138. https://doi.org/ 10.1016/j.rmed.2017.03.011

23. Kataria YP, Holter JF (1997) Immunology of sarcoidosis. Clin Chest Med 18:719-739. https://doi.org/10.1016/s0272-5231(05) 70415-9

24. Rømer FK, Sølling J (1981) Relationship between circulating immune complexes and angiotensin-converting enzyme in pulmonary sarcoidosis. Acta Med Scand 210:299-303. https://doi. org/10.1111/j.0954-6820.1981.tb09819.x

25. Saussine A, Tazi A, Feuillet S, Rybojad M, Juillard C, Bergeron A, Dessirier V, Bouhidel F, Janin A, Bensussan A, Bagot M, Bouaziz JD (2012) Active chronic sarcoidosis is characterized by increased transitional blood B cells, increased IL-10-producing regulatory B cells and high BAFF levels. PLoS ONE 7:e43588. https://doi.org/10.1371/journal.pone.004358 
26. Kudryavtsev I, Serebriakova M, Starshinova A et al (2020) Imbalance in B cell and $\mathrm{T}$ follicular helper cell subsets in pulmonary sarcoidosis. Sci Rep 10:1059. https://doi.org/10.1038/ s41598-020-57741-0

27. Weinberg I, Vasiliev L, Gotsman I (2000) Anti-dsDNA antibodies in sarcoidosis. Semin Arthritis Rheum 29:328-331. https:// doi.org/10.1016/s0049-0172(00)80019-0

28. Kobak S, Yilmaz H, Sever F, Duran A, Sen N, Karaarslan A (2014) The prevalence of antinuclear antibodies in patients with sarcoidosis. Autoimmune Dis 2014:351852. https://doi.org/10. $1155 / 2014 / 351852$

29. Kobak S, Ylmaz H, Sever F, Duran A, Sen N (2014) Anti-cyclic citrullinated peptide antibodies in patients with sarcoidosis. Sarcoidosis Vasc Diffuse Lung Dis 31:206-210

30. Maddrey WC, Johns CJ, Boitnott JK, Iber FL (1970) Sarcoidosis and chronic hepatic disease: a clinical and pathologic study of 20 patients. Medicine (Baltimore) 49:375-395. https://doi.org/ 10.1097/00005792-197009000-00002

31. Fagan EA, Moore-Gillon JC, Turner-Warwick M (1983) Multiorgan granulomas and mitochondrial antibodies. N Engl J Med 308:572-575. https://doi.org/10.1056/NEJM198303103081006

32. Stanca CM, Fiel MI, Allina J, Caracta CF, Odin JA (2005) Liver failure in an anti-mitochondrial antibody-positive patient with sarcoidosis: primary biliary cirrhosis or hepatic sarcoidosis? Semin Liver Dis 25:364-370. https://doi.org/https://doi.org/10. 1055/s-2005-916327

33. Kinloch AJ, Kaiser Y, Wolfgeher AJ, Eklund A, Clark MR, Grunewald J (2018) In situ humoral immunity to vimentin in HLA-DRB $1 * 03^{+}$patients with pulmonary sarcoidosis. Front Immunol 9:1516. https://doi.org/10.3389/fimmu.2018.01516

34. Baerlecken N, Pursche N, Witte T, Kniesch K, Höpfner M, Ernst D, Moosig F, Seeliger B, Prasse A (2020) Presence of antibodies binding to negative elongation factor $\mathrm{E}$ in sarcoidosis. J Clin Med 9:715. https://doi.org/10.3390/jcm9030715
35. Voortman M, Hendriks CMR, Elfferich MDP, Bonella F, Møller J, De Vries J, Costabel U, Drent M (2019) The burden of sarcoidosis symptoms from a patient perspective. Lung 197:155-161. https:// doi.org/10.1007/s00408-019-00206-7

36. Bakkers M, Merkies IS, Lauria G, Devigili G, Penza P, Lombardi R, Hermans MC, van Nes SI, De Baets M (2009) Faber CG (2009) Intra-epidermal nerve fiber density and its application in sarcoidosis. Neurology 73:1142-1148. https://doi.org/10.1212/ WNL.0b013e3181bacf05

37. Khan S, Zhou L (2012) Characterization of non-length-dependent small-fiber sensory neuropathy. Muscle Nerve 45:86-91. https:// doi.org/10.1002/mus.22255

38. Nakane S, Mukaino A, Maeda Y, Higuchi O, Matsuo H, Ando Y (2017) Extra-autonomic manifestations in autoimmune autonomic ganglionopathy: a Japanese survey. J Neurol Neurosurg Psychiatry 88:367-368. https://doi.org/10.1136/jnnp-2016-314707

39. Younger DS (2019) A postmortem study of a patient with low titer nicotinic acetylcholine receptor ganglionic antibody: implications for clinical neurologic disease. World J Neurosci 9:71-75. https:// doi.org/10.4236/wjns.2019.93005

40. Voorter CE, Drent M, Hoitsma E, Faber KG, van den BergLoonen EM (2005) Association of HLA DQB1 0602 in sarcoidosis patients with small fiber neuropathy. Sarcoidosis Vasc Diffuse Lung Dis 22:129-132. https://doi.org/10.1007/s11083-005-9013-x

41. Hoitsma E, Faber CG, van Kroonenburgh MJ, Gorgels AP, Halders SG, Heidendal GA, Kessels AG, Reulen JP, Drent M (2005) Association of small fiber neuropathy with cardiac sympathetic dysfunction in sarcoidosis. Sarcoidosis Vasc Diffuse Lung Dis 22:43-50

42. Smulders NM, Bast A, van Kroonenburgh MJ, Drent M (2008) Improvement of cardiac sympathetic nerve function in sarcoidosis. Sarcoidosis Vasc Diffuse Lung Dis 25:140-142 